\title{
The Scandinavian Total Ankle Replacement
}

\section{Survivorship at 5 and 8 Years Comparable to Other Series}

\author{
Alexia Karantana MD, Sally Hobson FRCS Orth, \\ Sunil Dhar FRCS Ed Orth
}

Published online: 16 July 2009

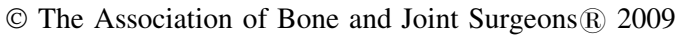

\begin{abstract}
Ankle arthroplasty is increasingly used to treat advanced ankle arthritis. Earlier prostheses have given way to second-generation implants, on which we are accumulating medium-term data. The Scandinavian Total Ankle Replacement (STAR) is a three-component uncemented implant in wide use in Europe and the only mobile-bearing prosthesis with conditional approval in the United States. We retrospectively reviewed 45 patients (52 ankles) who had primary total ankle replacements using STAR prostheses, in order to assess survivorship and add to the pool of clinical data provided by independent practitioners required to establish this treatment as a viable alternative to arthrodesis. The minimum followup was 60 months (range, 60-110 months). Clinical outcome was determined using the AOFAS score. We determined the rate of radiographic loosening and recorded complications and the need for further surgery. Survival was $90 \%$ (95\% CI 76.8 to 95.5 ) at 5 years and $84 \%$ (95\% CI 68.9 to 92.2 ) at 8 years. Six of 52 ankles $(11 \%)$ had component revision and two were
\end{abstract}

Each author certifies that he or she has no commercial associations (eg, consultancies, stock ownership, equity interest, patent/licensing arrangements, etc) that might pose a conflict of interest in connection with the submitted article.

Each author certifies that his or her institution has approved or waived approval for the human protocol for this investigation and that all investigations were conducted in conformity with ethical principles of research.

A. Karantana, S. Hobson, S. Dhar

Nottingham University Hospitals NHS Trust, Nottingham, UK

A. Karantana $(\bowtie)$

Division of Orthopaedic and Accident Surgery, Queens Medical

Centre, C Floor, West Block, Nottingham NG7-2UH, UK

e-mail: alexiak41@hotmail.com converted to fusion. The mean postoperative AOFAS score was 78 . The complication rate was $21 \%$. Subsequent surgery, excluding component revision, was performed in nine of $52(17 \%)$ ankles.

Level of Evidence: Level IV, therapeutic study. See the Guidelines for Authors for a complete description of levels of evidence.

\section{Introduction}

Interest in total ankle arthroplasty is increasing and many surgeons believe it is now a reasonable alternative to arthrodesis in selected patients with advanced degenerative disease. First-generation implants were cemented and either highly constrained, leading to early loosening at the cement bone interface, or completely unconstrained and therefore grossly unstable, leading to displacement and early failure. Results of ankle arthroplasty in the late 1980s were disappointing, with high failure rates. The fusion rate for failed ankle arthroplasty was reported as $36 \%$ by Kitaoka et al. at an average followup of 9 years [14]. Bolton-Maggs et al. fused $24 \%$ of arthroplasties at an average 5.5 years [2]. Second-generation designs have been mostly uncemented with either a fixed or mobile polyethylene bearing and better results [4]. Second-generation 5-year survival rates range from $78 \%$ to $93 \%$ and the 10-year survival from $76 \%$ to $80 \%[8,11,13,21]$. The STAR prosthesis (LINK® STAR Scandinavian Total Ankle Replacement, Waldemar Link, Hamburg, Germany) is one such second-generation, three-component mobile-bearing uncemented device currently in use in Europe and recently granted conditional approval by the FDA in the United States [18]. We have been using this implant since 1999. 
To confirm previous reports and add to the existing limited pool of medium-term clinical data provided by independent practitioners on this prosthesis, we (1) determined survivorship of the STAR prosthesis; (2) documented the reasons for revisions; (3) report the functional AOFAS scores; (4) report the followup radiographic appearance; (5) describe rates of major and minor complications; and (6) describe additional surgery performed without component revision.

\section{Patients and Methods}

We retrospectively reviewed all 45 patients (52 ankles) who underwent primary total ankle replacements using the STAR prosthesis (Fig. 1A-D) from March 1999 to December 2002. Seven patients had bilateral arthroplasties. There were $26(63 \%)$ men and 15 (37\%) women. The average age at surgery was 62 years (range, 33-81 years). In all patients total ankle arthroplasty was performed for
Fig. 1A-D (A-B) Preoperative and (C-D) 4-year postoperative AP and lateral weight bearing radiographic views of ankle arthrosis treated via the STAR prosthesis are shown. (A) Preoperative coronal deformity $\left(11^{\circ}\right)$ was measured with the angle of a line drawn along the superior talar dome relative to a line perpendicular to the mechanical axis of the tibia. (C) Measurement of varus or valgus of the prosthesis $\left(88^{\circ}\right)$ is shown. (D) Lateral radiograph at 4 years is shown.
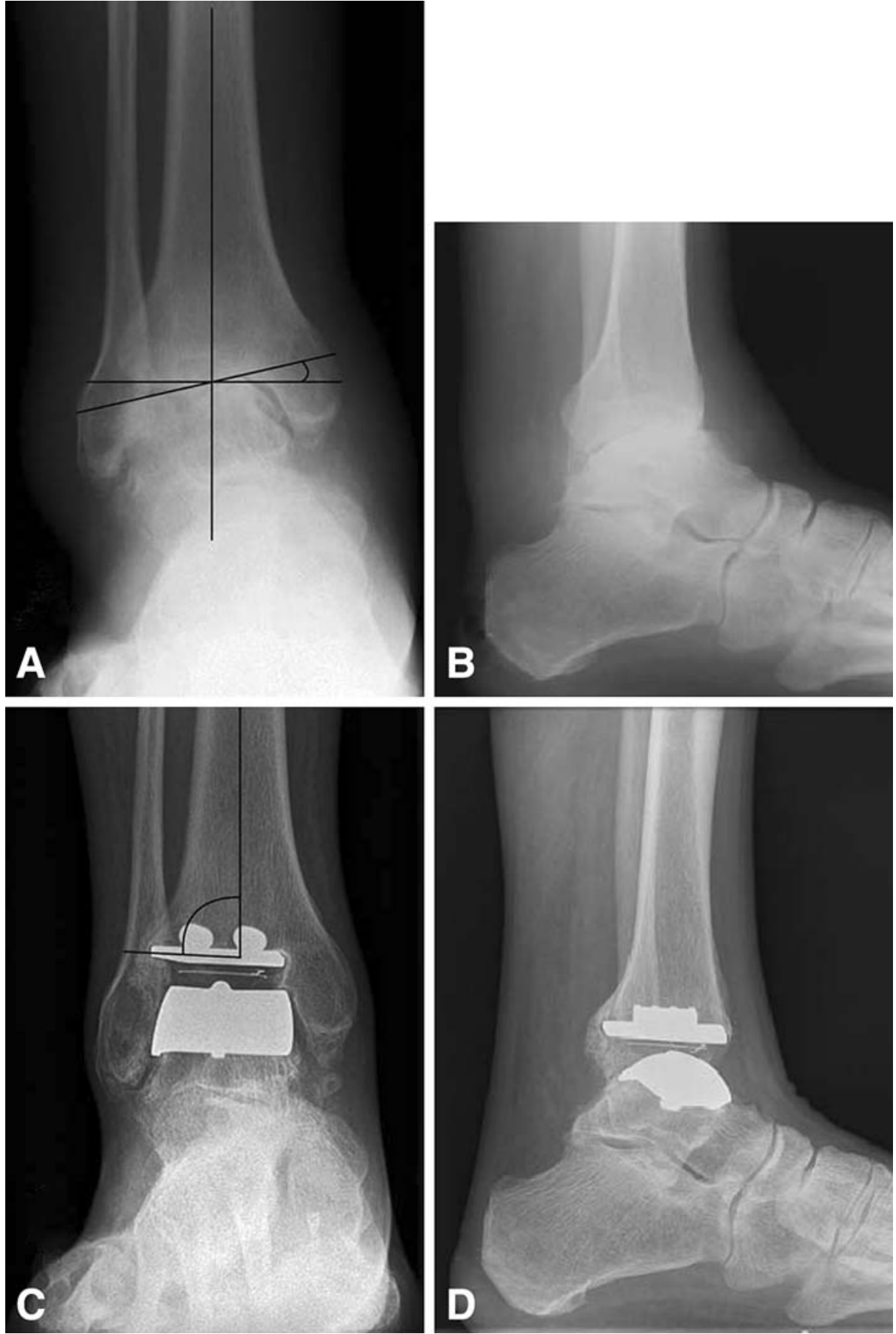
severe pain refractory to nonoperative management. The indication was osteoarthritis in $34(71 \%)$ ankles and inflammatory joint disease in 14 (29\%) ankles. Five of seven patients with bilateral disease had osteoarthritis and two had inflammatory arthropathy. Twelve patients with osteoarthritis had a documented history of a substantial injury to the ankle and we judged these to have secondary osteoarthritis. For the remaining 24 patients with osteoarthritis, there was no such history of trauma to the ankle joint. Thirteen (27\%) ankles had a preoperative coronal deformity at the tibiotalar joint of more than $10^{\circ}$, as measured radiographically on standardized, weight-bearing AP ankle radiographs (Fig. 1A-D). The majority of these ankles were in preoperative varus and the degree of deformity varied from $11^{\circ}$ to $25^{\circ}$ (Fig. 2). Four patients died of causes unrelated to the arthroplasty before completing the 5-year followup and were excluded from further analysis. None of these patients suffered complications nor underwent revision or other surgery to their ankle prior to death. The minimum followup for the living patients was 60 months (mean, 80 months; range, 60-110 months). None of these patients were lost to followup. Patients were informed that data collected would be used to determine outcome and informed consent was obtained.

The STAR prosthesis is an unconstrained, three-component mobile-bearing prosthesis. It has cobalt-chrome tibial and talar components, with a double coating of titanium and calcium phosphate, and an ultra-high-molecularweight polyethylene (UHMWPE) insert. It is implanted without cement.

All procedures were performed by the senior surgeon (SD) using a surgical technique previously described in detail [12]. Briefly the operation was performed through a standard anterior approach to the ankle via the bed of the tendons of either tibialis anterior or extensor hallucis longus. We thoroughly débrided the joint by excising the

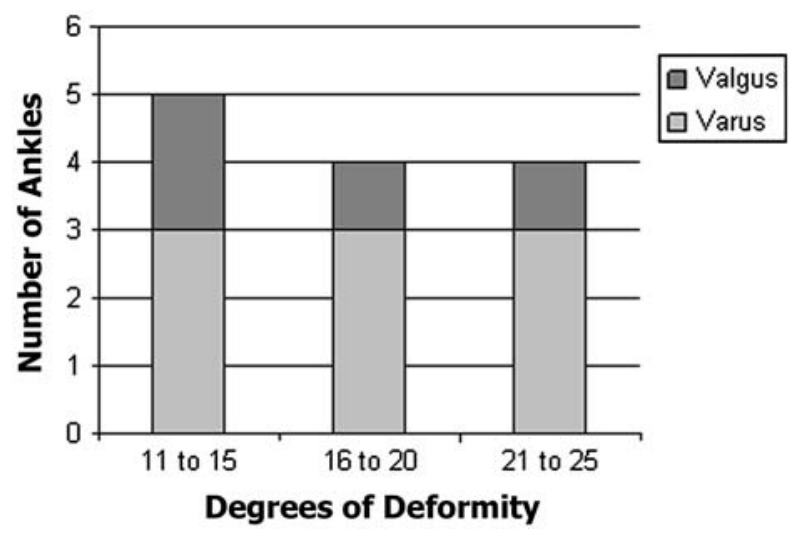

Fig. 2 Preoperative coronal deformity above $10^{\circ}$ at the tibiotalar joint was described in 13 ankles. This figure demonstrates the degree of deformity varying from $11^{\circ}$ to $25^{\circ}$ and also that the majority of these ankles were in preoperative varus. excess capsule, synovial tissue, and osteophytes. This frequently involved a thorough clearance of the medial and lateral gutters of all soft tissue and bony osteophytes. Often this resulted in "releasing" the talus sufficiently to correct any preexisting deformity passively. Our aim was to achieve a plantigrade foot with no residual varus, valgus, or equinus. Inability to do so was usually a result of either severe extraarticular soft tissue contracture, true bony deformity, or both. In the early years of the series, we typically accepted residual deformities at this stage and, once the implant had been inserted, corrected the hindfoot either by a calcaneal osteotomy or subtalar fusion, or both. These were carried out concomitantly or as staged procedures, depending on the complexity of the index operation. As we gained experience with the procedure, persisting varus after a thorough intraarticular soft tissue release was treated by additional extraarticular releases. These included a lengthening of the tibialis posterior tendon and a sequential release of the deltoid ligament, which in two patients was completely transected. If further correction was required, bony débridement was carried out in the medial gutter (for the more common varus deformity) to allow the talus to rotate into normal alignment. Occasionally, despite these measures, a hindfoot deformity persisted and was accepted, but corrected by a separate hindfoot procedure. Bony cuts were made using the standard jigging systems to insert the prostheses without bone cement following thorough pulsed lavage. At this stage, stability was assessed, and if there was any doubt about the integrity of the lateral ligaments, reconstruction of these structures was carried out. If there was residual equinus deformity, percutaneous (Hoke) lengthening of the tendo-Achilles was required. We closed the wounds in layers. At the end of the procedure a short-leg plaster back slab containing a foot pump (Orthofix AV Impulse System, Novamedix Services Ltd, Hampshire, England) was applied.

We administered prophylactic antibiotics (cefradine, $1 \mathrm{~g}$ ) at induction and a further three doses were administered postoperatively (cefradine, $500 \mathrm{mg}$ ), as is standard for arthroplasties on our unit. The plaster slab and foot pump was maintained for 24 hours. On the first to second postoperative day, the back slabs were changed to new full below-knee weight-bearing plaster casts and the patients were encouraged to mobilize with partial weight bearing with the help of walking aids. They were discharged when considered safe following physiotherapist and occupational therapist assessment. Patients were discharged on average on Day 4 postoperatively. At 2 weeks postoperatively wounds were checked and a short-leg walking cast was applied for a further 4 weeks.

At 6 weeks, the plaster was removed and patients were allowed to mobilize with full weight bearing out of plaster, having been taught range-of-movement exercises. They 
were advised to avoid high-impact and twisting and turning exercises; also that the postoperative swelling and stiffness would take several months to settle fully. They were taught simple calf stretching with a towel, dorsi/plantar flexion exercises while standing, and bilateral calf raises, and did not receive routine formal physiotherapy input.

Outpatient followup was planned for 2, 6, and 12 weeks. Patients were also seen at 6 months postoperatively and yearly thereafter. Clinical assessment included non-weightbearing range of movement at the ankle joint and assessment of hindfoot alignment. All measurements were made by a single independent assessor (KL) using a goniometer. Patients were assessed using the American Orthopaedic Foot and Ankle Surgeons' score [14] at most recent followup.

We obtained weight-bearing standing mortise AP and lateral views of the ankle joint (taken at $100 \mathrm{~cm}$ distance, exposure 65 kV, 1.5MAS, CR system) (Fig. 1). Sequential radiographs were assessed by a single observer $(\mathrm{SH})$ for the presence of aseptic loosening, defined as radiolucency greater than $2 \mathrm{~mm}$ in width, cavitation at the tibial component interface or migration of the components [20]. The shape of the talar component hides the bone interface from view and only migration can be assessed. Component migration was assessed through comparison of prosthesis alignment on sequential radiographs. Implant alignment was assessed through angular measurements of (1) the angle between the anatomical axis of the tibia and the articular surface of the tibial implant on the AP radiograph; (2) the angle between the anatomical axis of the tibia and the articular surface of the tibial component on the lateral radiograph; and (3) the angle formed by the talar component and the talar neck. This is the method described for the STAR implant in great detail by Wood and Deakin [20].

Survivorship analysis was performed using the KaplanMeier survivorship method and life table analysis [3] with the need for revision by exchange or removal of any part of the prosthesis or conversion to arthrodesis as the end point.

\section{Results}

Prosthesis survival was $90 \%$ at 5 years (95\% CI 76.8 to 95.5 ) and $84 \%$ at 8 years (95\% CI 68.9 to 92.2) (Table 1) (Fig. 3).

Eight patients had revision surgery (Table 2). Six had component revision and two underwent nail fusion. The average time to revision was 44 months (range, 3100 months). In two patients, the revision was secondary to tibial stress fracture. In a further two, we revised for insert fracture due to edge loading. Two revision procedures were performed for stiffness associated with pain. This stiffness resolved by revision of the tibial component in one patient;
Table 1. Kaplan-Meier life-table survival of 48 total ankle replacements with the decision to revise as the endpoint

\begin{tabular}{llllll}
\hline $\begin{array}{l}\text { Years since } \\
\text { operation }\end{array}$ & $\begin{array}{l}\text { Number } \\
\text { at start }\end{array}$ & $\begin{array}{l}\text { Number } \\
\text { revised }\end{array}$ & Lost & $\begin{array}{l}\text { Survival } \\
\text { rate }(\%)\end{array}$ & $\begin{array}{l}95 \% \text { Confidence } \\
\text { interval }\end{array}$ \\
\hline $0-1$ & 48 & 2 & 0 & 96 & 84.3 to 98.9 \\
$1-2$ & 46 & 1 & 0 & 94 & 81.9 to 97.9 \\
$3-4$ & 45 & 1 & 0 & 92 & 79.3 to 96.8 \\
$4-5$ & 44 & 1 & 0 & 90 & 76.8 to 95.5 \\
$5-6$ & 43 & 1 & 8 & 87 & 73.8 to 94.1 \\
$6-7$ & 34 & 1 & 15 & 84 & 68.9 to 92.2 \\
$7-8$ & 18 & 0 & 12 & 84 & 68.9 to 92.2 \\
$8-9 *$ & 6 & 1 & 3 & 65 & 24.3 to 88.0 \\
\hline
\end{tabular}

* Two patients had a followup of 9 to 9.2 years and no failures.

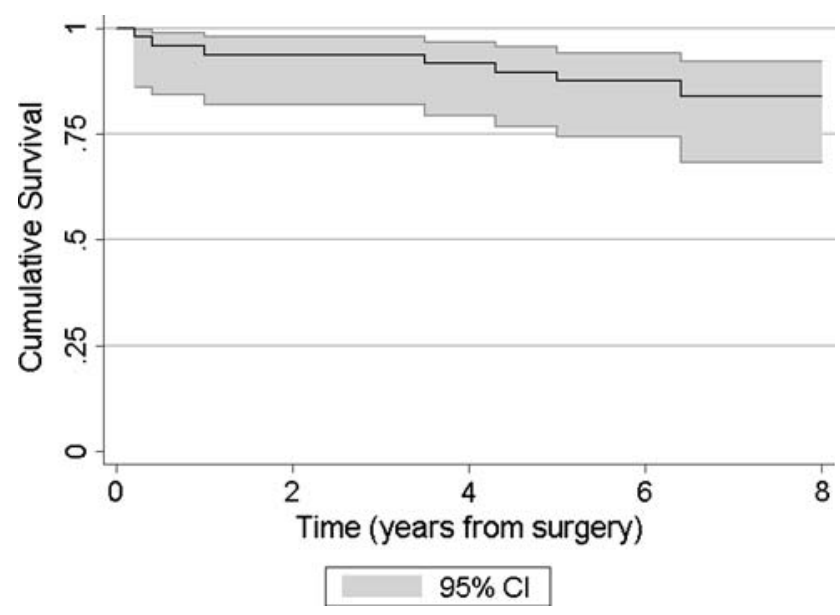

Fig. 3 Kaplan-Meier survival curve (with 95\% confidence interval in shading) for STAR total ankle replacement in this series is shown, with the need for revision by exchange or removal of any part of the prosthesis or conversion to arthrodesis as the end point. Survival was $90 \%$ at 5 years and $84 \%$ at 8 years.

the other developed aseptic loosening of the tibial component with a linear lucency of more than $2 \mathrm{~mm}$ along the bony interface and migration of the component into valgus, for which we performed fusion. One patient developed progressive talar collapse so that the talar component eventually came to rest virtually on the calcaneus. Of the six component revision patients, one underwent further revision to fusion at a later date as a result of infection. This was the only case of deep infection, albeit secondary, in the series. Fusions were performed using a cannulated intramedullary nail (Ace ${ }^{\mathrm{TM}}$ VersaNail tibial nail; DePuy, Warsaw, Ind.). Bony apposition was achieved in one case through $1 \mathrm{~cm}$ shortening, without bone graft. In the other, distal fibular autograft and femoral head allograft were used. Patients were treated non-weight bearing in a cast for 6 weeks and fully weight bearing in an Aircast $\AA$ (DJO Incorporated, Vista, CA) walker for a further 6 weeks. 
Table 2. Mode of failure, time to revision and revision type for cases of failed total ankle replacement

\begin{tabular}{lllllll}
\hline Case & Sex & Age & $\begin{array}{l}\text { Time to failure } \\
\text { (months) }\end{array}$ & Indication & Mode of failure & Revision type \\
\hline 1 & M & 60 & 3 & Osteoarthritis & Stress fracture & Tibial component + insert \\
2 & M & 33 & 5 & Inflammatory & Stress fracture & Tibial component \\
3 & M & 62 & 12 & Osteoarthritis & Stiffness + pain & Tibial component \\
4 & M & 51 & 42 & Secondary osteoarthritis & Stiffness & Insert \\
5 & F & 56 & 52 & Secondary osteoarthritis & Insert fracture due to edge loading & Talar component + insert \\
6 & M & 64 & 60 & Osteoarthritis & Insert fracture due to edge loading & Insert \\
7 & F & 75 & 77 & Osteoarthritis & Talar subsidence & Fusion \\
8 & M & 37 & 100 & Inflammatory & Loosening & Fusion \\
\hline
\end{tabular}

The mean AOFAS score at last followup was $78 \pm 18$ (range, 30-100). The mean arc of dorsoplantar flexion was $23^{\circ} \pm 12^{\circ}$ (range, $0-55^{\circ}$ ). Correction of preoperative hindfoot deformity, as measured clinically, was achieved to within $5^{\circ}$ of neutral in all but four of 13 ankles.

Progressive radiographic loosening was observed in one patient who, as mentioned above, underwent revision for loosening of the tibial component. There were no other cases of progressive lucency or of component migration. Three patients had asymptomatic nonprogressive 2-mm lucent lines visible behind the tibial component, which required no further intervention. Minor bone growth around the tibial component was common but we only had one patient with large heterotopic ossification posteriorly. This was removed at revision surgery and helped improve range of movement.

There were 11 postoperative complications in 10 of 48 ankles $(21 \%)$. Three were superficial wound infections in the early postoperative period, which resolved with antibiotic therapy (intravenous and/or oral flucloxacillin, $500 \mathrm{mg}$ once a day, for a period of 1 to 2 weeks as required). There were no primary deep infections. No cases of venous thromboembolism occurred. The other eight complications were fractures $(8 / 48$ or $17 \%)$. Five were intraoperative, one medial, and four of the lateral malleolus; these fractures were undisplaced and all united after a period of protected weight bearing. There were also three postoperative fractures, two of which led to revision, as above described.

Subsequent surgery, excluding component revision, was performed in nine of 48 patients (19\%). Five underwent calcaneal osteotomies to correct painful varus or valgus deformity attributable to the subtalar joint. Three were performed to correct residual deformity in patients with preoperative hindfoot deformity. None have since failed. An additional patient underwent subtalar fusion for degenerative disease and one had a lateral malleolar cyst successfully grafted. Two ankles underwent arthroscopic débridement for impingement-type pain over the medial gutter. These were performed at 4 months in the first case and 39 months after arthroplasty in the second. They were carried out under distraction using standard anteromedial and anterolateral portals, taking extreme care not to scratch the metal components. In the first case, symptoms improved following the procedure. In the second, there was no improvement; the patient underwent successful calcaneal osteotomy 9 months later.

\section{Discussion}

Interest in total ankle arthroplasty as a surgical alternative to arthrodesis has grown over the last few years. Firstgeneration, two-component constrained designs have been succeeded by second-generation three-component minimally or nonconstrained implants. Though longer followup is necessary, mid-term studies of second-generation total ankle arthroplasty suggest higher survival that for firstgeneration implants (Table 3). The STAR ankle prosthesis is such an implant. It is in wide use in Europe and has received conditional FDA approval in the United States, pending clinical studies to evaluate long-term safety and performance [18]. Five year survival of the STAR has been reported as high as $93 \%$ and 8 year survival as $88 \%$ in the largest single series published by Wood [21]. To confirm published reports we (1) determined survivorship of the STAR prosthesis; (2) documented the reasons for revisions; (3) report the functional AOFAS scores; (4) report the followup radiographic appearance; (5) describe rates of complications; and (6) describe additional surgery performed without component revision.

We note some limitations of our study. First, we did not have preoperative AOFAS scoring for all patients to quantify net clinical gain. Second, there is no standardized method to clinically measure range of movement of the ankle after arthroplasty. Measurements of motion and hindfoot deformity inevitably reflect the combined tibiotalar and subtalar joints. Third, the STAR talar component 
conceals the bone-implant interface, it is possible that some cases of talar loosening may have remained undetected [21]. Fourth, the number of failures in our study was too small to allow statistical exploration of any causal relationships: all but three patients had well-functioning prostheses in situ at a minimum of 5 years. The remaining three underwent successful fusion, which is an effective salvage procedure after failed total ankle arthroplasty [5]. This corresponds to a fusion rate of $6 \%$ (3/48), which is comparable to the $5 \%(29 / 572)$ conversion rate to fusion reported in a recent meta-analysis of total ankle arthroplasty [9].

We observed survival of $90 \%$ at 5 years and $84 \%$ at 8 years with a revision rate of $17 \%$. These figures are comparable to other series of the STAR in particular $[1,21]$ and ankle replacement prostheses in general $[8,11,13]$ (Table 3). The above-mentioned meta-analysis quoted a 5 -year survival of $78 \%$ and a 10 -year survival rate of $77 \%$ for ankle arthroplasty, with an overall revision rate of $7 \%$ [9]. We suspect the survival figure will continue to improve with better case selection, attention to operative detail and correction of any residual deformity or instability.

The clinical outcome in terms of the American Orthopaedic Foot and Ankle Surgeons' score and range of motion data were comparable both with published series of the STAR implant $[1,21]$ and the results of meta-analysis of combined data [9]. It is our observation that the rehabilitation time following surgery is prolonged with slow but progressive improvement in the range of movement and reduction in discomfort and swelling. This usually occurs over 12 months.

Incidence of loosening and component migration leading to revision was low compared to published literature (Table 3). While this may reflect the unconstrained design of the implant and also possibly the improved bony interface achieved through dual titanium calcium phosphate coating that was not in use in older designs, we believe our assiduous attempts at correcting residual hindfoot deformity and instability also contribute considerably towards this. Asymptomatic lucencies around the tibial component were also uncommon in our series. However, the STAR talar component does conceal the body of the talus on postoperative radiographs [21]; so it is possible that some cases of asymptomatic loosening in this area remained undetected.

Complications in our study in terms of wound healing, infection, and heterotopic ossification were low [19, 21]. The overall fracture rate of $17 \%$ (8/48) was comparable to the literature with reports of fracture incidence of $20 \%$ [16]. Intraoperative fractures all healed without the need for further intervention, contrary to reports that these need to be operatively stabilized [16]. While we had insufficient data to examine the relationship between fracture and a 
learning curve, we suspect that in arthroplasties performed in the later years of this series (since 2002), fractures were less frequent, as suggested by other authors [6, 15-17].

A substantial proportion of patients (19\%) in this series underwent further surgery not involving revision of the prosthesis. Though reoperation rates are not routinely reported alongside survival data, this figure is comparable to the $22 \%$ reoperation rate quoted by Hosman et al. [13]. Interventions were successful, however, and none of these ankles went on to fail. Further surgery can also be reduced by proper case selection (ie, little or no deformity). However, in severely deformed ankles, a staged approach is more logical, where total ankle arthroplasty is carried out in the first instance correcting ankle deformity, and subsequent hindfoot and soft tissue procedures are carried out to correct residual deformities or instability. This series includes $27 \%$ of cases with a preoperative hindfoot deformity of more than $10^{\circ}$. The presence of substantial preoperative varus or valgus deformity in ankle arthroplasty remains a topic of debate. Early reports suggested that realignment procedures at the time of primary ankle replacement can be performed safely [17] and that deformity correction can be maintained in the short term [10]. Other authors experienced poor results in the presence of deformity [7, 21]. We have recently published a large study focusing directly on the subject of deformity [12]. We compared patients with a preoperative hindfoot deformity under $10^{\circ}$ to a group with greater deformity $\left(11^{\circ}-30^{\circ}\right)$ and found no difference in range of motion, complication, and survival rate, whereas patients with greater deformity had better postoperative AOFAS scores. Further studies and experience on the subject of deformity will be required before arthroplasty in the presence of severe deformity becomes common practice. We believe, however, that hindfoot deformity should not be an absolute contraindication to total ankle arthroplasty, as long as additional realignment procedures, such as soft tissue releases, ligament reconstructions, calcaneal osteotomy, and subtalar fusion, are performed as and when necessary.

Our data confirm reports that the dual-coated STAR implant has acceptable survivorship in the mid-term, though additional procedures will be necessary in some patients. As experience with total ankle arthroplasty increases, we expect to see survival rates increase further to become comparable to other long-established joint replacements such as the hip and knee. However, we believe care needs to be taken to ensure correct alignment of the implant, as well as satisfactory ligament balancing and stability and correction of any residual deformity.

Acknowledgments We thank Katie Lee, senior physiotherapist at the Queens Medical Centre, for providing independent assessor outcome data for this study.

\section{References}

1. Anderson T, Montgomery F, Carlsson A. Uncemented STAR total ankle prostheses. Three to eight-year follow-up of fifty-one consecutive ankles. J Bone Joint Surg Am. 2003;85:1321-1329.

2. Bolton-Maggs BG, Sudlow RA, Freeman MA. Total ankle arthroplasty. A long-term review of the London Hospital experience. J Bone Joint Surg Br. 1985;67:785-790.

3. Collett D. Modeling Survival Data in Medical Research, 2nd ed. Boca Raton, FL: Chapman \& Hall/CRC; 2003.

4. Cracchiolo A 3rd, Deorio JK. Design features of current total ankle replacements: implants and instrumentation. J Am Acad Orthop Surg. 2008;16:530-540.

5. Culpan P, Le Strat V, Piriou P, Judet T. Arthrodesis after failed total ankle replacement. J Bone Joint Surg Br. 2007;89:11781183.

6. Deorio JK, Easley ME. Total ankle arthroplasty. Instr Course Lect. 2008;5:383-413.

7. Doets HC, Brand R, Nelissen RG. Total ankle arthroplasty in inflammatory joint disease with use of two mobile-bearing designs. J Bone Joint Surg Am. 2006;88:1272-1284.

8. Fevang BT, Lie SA, Havelin LI, Brun JG, Skredderstuen A, Furnes O. 257 ankle arthroplasties performed in Norway between 1994 and 2005. Acta Orthop. 2007;78:575-583.

9. Haddad SL, Coetzee JC, Estok R, Fahrbach K, Banel D, Nalysnyk L. Intermediate and long-term outcomes of total ankle arthroplasty and ankle arthrodesis. A systematic review of the literature. J Bone Joint Surg Am. 2007;89:1899-1905.

10. Haskell A, Mann RA. Ankle arthroplasty with preoperative coronal plane deformity: short-term results. Clin Orthop Relat Res. 2004;424:98-103.

11. Henricson A, Skoog A, Carlsson A. The Swedish Ankle Arthroplasty Register: an analysis of 531 arthroplasties between 1993 and 2005. Acta Orthop. 2007;78:569-574.

12. Hobson SA, Karantana A, Dhar S. Total ankle replacement in patients with significant pre-operative deformity of the hindfoot. J Bone Joint Surg Br. 2009;91:481-486.

13. Hosman AH, Mason RB, Hobbs T, Rothwell AG. A New Zealand national joint registry review of 202 total ankle replacements followed for up to 6 years. Acta Orthop. 2007;78:584-591.

14. Kitaoka HB, Alexander IJ, Adelaar RS, Nunley JA, Myerson MS, Sanders M. Clinical rating systems for the ankle-hindfoot, midfoot, hallux, and lesser toes. Foot Ankle Int. 1994;15:349353.

15. Kumar A, Dhar S. Total ankle replacement: early results during learning period. Foot Ankle Surg. 2007;13:19-23.

16. McGarvey WC, Clanton TO, Lunz D. Malleolar fracture after total ankle arthroplasty: a comparison of two designs. Clin Orthop Relat Res. 2004;424:104-110.

17. Schuberth JM, Patel S, Zarutsky E. Perioperative complications of the Agility total ankle replacement in 50 initial, consecutive cases. J Foot Ankle Surg. 2006;45:139-146.

18. Summary of the Orthopaedic and Rehabilitation Devices Panel Meeting - April 24, 2007. Available at http://www.fda.org/cdrh/ panel/summary/ortho-042407.html. Accessed November 23, 2008.

19. Valderrabano V, Hintermann B, Dick W. Scandinavian total ankle replacement: a 3.7-year average followup of 65 patients. Clin Orthop Relat Res. 2004;424:47-56.

20. Wood PL, Deakin S. Total ankle replacement. The results in 200 ankles. J Bone Joint Surg Br. 2003;85:334-341.

21. Wood PL, Prem H, Sutton C. Total ankle replacement: mediumterm results in 200 Scandinavian total ankle replacements. J Bone Joint Surg Br. 2008;90:605-609. 